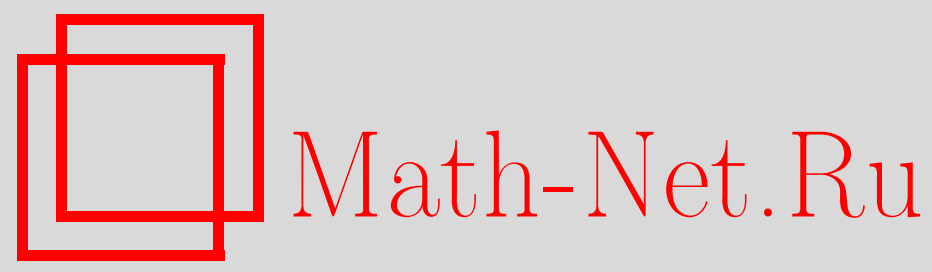

Ю. Г. Гераськина, О стартовых состояниях автоматной модели легких в чистой среде, Дискрет. матем., 2008, том 20, выпуск 3, 119-135

DOI: https://doi.org/10.4213/dm1019

Использование Общероссийского математического портала Math-Net.Ru подразумевает, что вы прочитали и согласны с пользовательским соглашением http://www . mathnet.ru/rus/agreement

Параметры загрузки:

IP: 35.173 .219 .12

26 апреля 2023 г., $16: 46: 26$ 


\title{
О стартовых состояниях автоматной модели легких в чистой среде
}

\author{
() 2008 г. Ю. Г. Гераськина
}

\begin{abstract}
В работе продолжается изучение функционирования легких человека. Ранее была построена автоматная модель самоочищения запыленных легких в чистой среде. В предлагаемой работе проводится изучение диаграммы Мура соответствующего автомата. В качестве характерных состояний диаграммы выбраны состояния, у которых нет предшественников, такие состояния называются стартовыми. В работе изучаются стартовые состояния диаграммы Мура.
\end{abstract}

\section{1. Введение}

В предлагаемой работе продолжается изучение функционирования легких человека, начатое в $[2,3,4]$. В качестве главной функции легких рассматривается свойство их самоочищения как от внутреннего секрета, так и от поступающего извне вещества в легкие. В [2] установлено, что процесс самоочищения легких можно моделировать с помощью автоматов. Здесь мы изучаем свойства таких автоматов.

Легкие образуют древовидную структуру бронхов, в которых имеются реснички, играющие роль эскалаторного механизма вывода вещества во внешнюю среду. Бронхи имеют разные пропускные способности и разную эффективность ресничек. Чем выше от самых мелких бронхов, тем мощнее механизм передачи вещества изнутри вовне.

Предполагается, что в момент $t$ распределено некоторое количество вещества по ресничкам легких. Тогда в момент $t+1$ по определенным правилам происходит перемещение вещества в легких с помощью ресничек по направлению к трахее. Этот процесс продолжается до полного освобождения легких от этого вещества.

Возникает задача построения модели легочного механизма самоочищения как в условиях чистой среды, так и в условиях возможного запыления легких из среды в процессе дыхания, а также задача изучения свойств этой модели.

Ранее автором была построена такая модель процесса самоочищения запыленных легких в предположении, что они функционируют в чистой среде [3]. Как отмечено выше, автором было показано, что такая модель является автоматной, и потому разные свойства процесса самоочищения могут быть исследованы с помощью изучения соответствующих автоматов. Основной характеристикой автоматов, как известно [1], является его диаграмма Мура, построение и изучение свойств которой для модели легких означало бы установление свойств процесса их самоочищения. В предлагаемой работе проводится изучение этой диаграммы Мура. В качестве характерных состояний диаграммы выбраны те состояния, у которых нет предшественников. Такие состояния называются стартовыми. 
Основными результатами предлагаемой работы являются критериальное описание стартовых состояний и нахождение их числа, а также нахождение числа всех состояний указанной диаграммы.

\section{2. Основные понятия, постановка задач и результаты}

Представим легкие полным дихотомическим ориентированным к корню деревом, которое будем называть $I$-деревом и обозначать $D^{-1}$, со следующими параметрами.

Пусть $\mathbf{N}-$ множество натуральных чисел, $\mathbf{N}_{0}=\{0\} \cup \mathbf{N}$ и $l, l \in \mathbf{N}$, будем обозначать глубину этого $I$-дерева. Полагаем, что ребро $I$-дерева $D^{-1}$, инцидентное корню, имеет глубину 1.

Каждое ребро $D^{-1}$ разделено на $n, n \in \mathbf{N}$, равных частей, называемых ресничками, и занумерованных числами $i, i=1,2, \ldots, n$, возрастающими в направлении, обратном ориентации ребра.

Припишем каждому ребру глубины $j, j=1,2, \ldots, l$, два числа $2^{l-j} b$ и $2^{l-j} r$, где $b, r \in \mathbf{N}$ и $r \leqslant b$, называемых максимальной нагрузкой и мерой переброса ресничек ребер глубины $j$, соответственно.

Такое $I$-дерево $D^{-1}$ с параметрами $b, r, n$ и $l$ обозначим $D^{-1}(b, r, n, l)$. Свяжем с ним некоторый процесс, который назовем процессом дыхания. Он обусловлен рядом допущений. Считаем, что в $D^{-1}(b, r, n, l)$ заданы распределения значений нагрузок по всем ресничкам, учитывая, что нагрузка может быть нулевой. Пусть $V^{\prime}-$ суммарная нагрузка по всем ресничкам, а $V$ - максимально возможная суммарная нагрузка по всем ресничкам. Нагрузку $V$ назовем объемом $I$-дерева (легких), а $V^{\prime}-$ исходным объемом загруженности $I$-дерева. $I$-дерево $D^{-1}(b, r, n, l)$ с исходным объемом загруженности $V^{\prime}$ обозначим $D^{-1}\left(b, r, n, l ; V^{\prime}\right)$.

Каждая ресничка осуществляет прием вещества извне и переброс своей нагрузки на следующую ресничку с меньшим номером внутри ребра. Прием ресничкой вещества, имеющего массу $d, d \in \mathbf{N}_{0}, d \leqslant V-V^{\prime}$, из внешней среды внутри ребра осуществляется по следующему правилу (для этого правила ориентация считается обратной к заданной).

$\left(\mathrm{A}_{1}\right)$ Если ресничка имеет максимальную нагрузку, то прием вещества не осуществляется.

(Б 1$)$ При немаксимальной нагрузке $d_{1}$ первой такой реснички она осуществляет прием вещества максимально возможной массы $d_{2}$ такой, что $d_{1}+d_{2} \leqslant \min (b, d)$, где $b$ - максимальная нагрузка этой реснички.

$\left(\mathrm{B}_{1}\right)$ Следующая за ресничкой из $\left(\mathrm{Б}_{1}\right)$ принимает массу $d_{3}$, как и в $\left(\mathrm{Б}_{1}\right)$, с заменой там $d$ на $d-d_{2}$.

$\left(\Gamma_{1}\right)$ Оставшаяся масса вещества опускается до следующей реснички с большим номером в ребре, для которой не выполняется условие $\left(\mathrm{A}_{1}\right)$. Она осуществляет прием вещества по правилу $\left(\mathrm{B}_{1}\right)$ или $\left(\mathrm{Б}_{1}\right)$.

(Д 1 ) Если ресничка в рассматриваемом ребре является последней, не удовлетворяющей условию $\left(\mathrm{A}_{1}\right)$, то оставшаяся масса вещества делится пополам (если число нечетное, то одна из частей на единицу больше другой), и каждая из частей вещества воспринимается соответствующими ребрами, как описано выше.

$\left(\mathrm{E}_{1}\right)$ Процесс, описываемый позициями $\left(\mathrm{A}_{1}\right)-\left(Д_{1}\right)$, начинается с ребра, которое инцидентно корню. 
Переброс ресничкой вещества осуществляется на следующую ресничку с меньшим номером внутри ребра по такому правилу.

$\left(\mathrm{A}_{2}\right)$ Если следующая ресничка имеет ненулевую нагрузку, то переброс с реснички не осуществляется.

$\left(Б_{2}\right)$ Если нагрузка реснички не превосходит $r$, где $r$ - ее мера переброса, и не выполнено условие $\left(\mathrm{A}_{2}\right)$, то перебрасывается на следующую вся нагрузка реснички и считается, что ее нагрузка становится равной нулю.

$\left(\mathrm{B}_{2}\right)$ Если на ресничке нагрузка $m$ и $m>r$, то она перебрасывает на следующую ресничку нагрузку $r$ и оставляет у себя нагрузку $m-r$. Если ресничка в ребре последняя, то переброс нагрузки осуществляется по правилам $\left(\mathrm{A}_{2}\right),\left(\mathrm{Б}_{2}\right),\left(\mathrm{B}_{2}\right)$.

$\left(\Gamma_{2}\right)$ Если ребро инцидентно корню, то переброс с наименьшей по номеру реснички осуществляется в среду по правилам $\left(\mathrm{b}_{2}\right)$ и $\left(\mathrm{B}_{2}\right)$ в предположении, что среда играет роль реснички с нулевой нагрузкой.

(Д2) Если ребро не инцидентно корню, то есть его вершина инцидентна следующему ребру, то нагрузка с наименьшей по номеру реснички этого ребра передается наибольшей по номеру ресничке другого ребра по правилам $\left(\mathrm{A}_{2}\right),\left(\mathrm{Б}_{2}\right),\left(\mathrm{B}_{2}\right)$.

Считаем, что процесс дыхания осуществляется в дискретные моменты времени $t=1,2, \ldots$ В первый момент $I$-дерево $D^{-1}\left(b, r, n, l ; V^{\prime}\right)$ имеет заданное распределение нагрузок по его ресничкам. Ко второму моменту осуществляется прием вещества массой $d(1)$ по правилам $\left(\mathrm{A}_{1}\right)-\left(\mathrm{E}_{1}\right)$, и затем осуществляется переброс нагрузок с реснички на ресничку во всем $I$-дереве или выброс в среду в соответствии с правилами $\left(\mathrm{A}_{2}\right)-\left(Д_{2}\right)$. Если в легкие подается масса $d$, не превосходящая объема легких, то та ее часть, которая не осела на ресничках, выбрасывается в среду.

Другими словами, за один момент (шаг) происходит вдох и выдох.

Если в каждый момент $t=1,2, \ldots$ все реснички $I$-дерева $D^{-1}\left(b, r, n, l ; V^{\prime}\right)$ осуществляют прием вещества нулевой массы, то такой процесс называется процессом самоочищения этого $I$-дерева. Процесс самоочищения заканчивается в такой момент $t$, в котором нагрузки всех ресничек $I$-дерева $D^{-1}\left(b, r, n, l ; V^{\prime}\right)$ впервые стали равными нулю.

Под распределением нагрузки $V^{\prime} I$-дерева $D^{-1}\left(b, r, n, l ; V^{\prime}\right)$ будем понимать любое из возможных распределений нагрузок всех его ресничек такое, что суммарный объем их нагрузок равен $V^{\prime}$. Ясно, что $V^{\prime} \leqslant V$, где $V$ - объем $I$-дерева $D^{-1}\left(b, r, n, l ; V^{\prime}\right)$ и $V=2^{l-1} \mathrm{bnl}$. Такие распределения будем называть конфигурациями нагрузки $V^{\prime}$ по ресничкам $I$-дерева $D^{-1}(b, r, n, l)$.

Занумеруем все реснички $I$-дерева $D^{-1}(b, r, n, l)$ таким образом, что ресничка с номером $i j k$ является $k$-й ресничкой $j$-го ребра глубины $i$, где $1 \leqslant i<l, 1 \leqslant j \leqslant 2^{i-1}$, $1 \leqslant k \leqslant n$, а нумерация ребер одной глубины идет слева направо. Тогда в каждый момент $t$ конфигурацию нагрузки $V^{\prime}(t)$ в $I$-дереве $D^{-1}(b, r, n, l)$ можно задать набором

$$
q(t)=\left(q_{111}(t), q_{112}(t), \ldots, q_{i j k}(t), \ldots, q_{l 2^{l-1} n}(t)\right),
$$

в котором каждая координата $q_{i j k}(t)$ равна нагрузке реснички с номером $i j k$ в момент $t$, причем

$$
0 \leqslant q_{i j k}(t) \leqslant 2^{l-i} b, \quad \sum_{111}^{l 2^{l-1} n} q_{i j k}(t)=V^{\prime}(t) .
$$


Пусть в процессе самоочищения конфигурации нагрузки $V^{\prime}(t)$ в каждый момент $t$ изменяются по правилам $\left(\mathrm{A}_{2}\right)-\left(\right.$ Д$\left._{2}\right)$. Тогда процесс самоочищения можно представить некоторым конечным автономным автоматом $A(b, r, n, l)$ с одним финальным состоянием, что было сделано автором ранее в [2]. Состояниями построенного автомата являются конфигурации нагрузок $V^{\prime}(t)$ в $I$-дереве $D^{-1}(b, r, n, l)$, которые называем также состояниями этого $I$-дерева, а законы перехода из одного состояния в другое считаем указанными выше. Отметим, что понятие состояния $I$-дерева не зависит от параметра $r$, а полностью определяется параметрами $b, n, l$ и распределением вещества по этому $I$-дереву.

Учитывая отмеченную независимость понятия состояния $I$-дерева от $r$, обозначим через $Q(b, n, l)$ множество состояний $I$-дерева $D^{-1}(b, r, n, l)$, а его мощность через $|Q(b, n, l)|$, полагая далее, что $n>1$.

Возникает задача нахождения величины $|Q(b, n, l)|$, решение которой содержит следующее утверждение.

Теорема 1. Справедливо равенство

$$
|Q(b, n, l)|=\prod_{i=1}^{l}\left(2^{l-i} b+1\right)^{2^{i-1} n} .
$$

Следствие 1. Справедливы оценки

$$
b^{\left(2^{l}-1\right) n} 2^{\left(2^{l}-l-1\right) n}<|Q(b, n, l)| \leqslant(b+1)^{\left(2^{l}-1\right) n} 2^{\left(2^{l}-l-1\right) n} .
$$

Доказательство. Ясно, что

$$
\prod_{i=1}^{l}\left(2^{l-i} b+1\right)^{2^{i-1} n}=\prod_{i=1}^{l}\left(2^{l-i}\left(b+1 / 2^{l-i}\right)\right)^{2^{i-1} n} .
$$

Далее, при $i=1,2, \ldots, l$ выполняются неравенства $0<1 / 2^{l-i} \leqslant 1$. Следовательно, с учетом теоремы 1

$$
\begin{aligned}
|Q(b, n, l)|> & \prod_{i=1}^{l}\left(2^{l-i} b\right)^{2^{i-1} n}=2^{n \sum_{i=1}^{l}(l-i) 2^{i-1}} b^{n \sum_{i=1}^{l} 2^{i-1}}=2^{\left(2^{l}-l-1\right) n} b^{\left(2^{l}-1\right) n} \\
|Q(b, n, l)| & \leqslant \prod_{i=1}^{l}\left(2^{l-i}(b+1)\right)^{2^{i-1} n}=2^{n \sum_{i=1}^{l}(l-i) 2^{i-1}}(b+1)^{n \sum_{i=1}^{l} 2^{i-1}} \\
& =2^{\left(2^{l}-l-1\right) n}(b+1)^{\left(2^{l}-1\right) n} .
\end{aligned}
$$

Таким образом,

$$
b^{\left(2^{l}-1\right) n} 2^{\left(2^{l}-l-1\right) n}<|Q(b, n, l)| \leqslant(b+1)^{\left(2^{l}-1\right) n} 2^{\left(2^{l}-l-1\right) n} .
$$

Следствие доказано.

Следствие 2. При $l \rightarrow \infty$

$$
\log _{2}|Q(b, n, l)| \asymp 2^{l} .
$$


Справедливость следствия устанавливается логарифмированием левой и правой частей неравенств из следствия 1.

Заметим, что диаграмма Мура для автомата $A(b, r, n, l)$ образует частичный порядок по отношению к переходу от одного состояния в другое и допущением того, что финальное состояние никуда не переходит. Тогда последнее является наименьшим элементом этого порядка, в нем есть максимальные элементы и нет наибольшего элемента. Максимальный элемент этого частичного порядка называем стартовым состоянием. Оно характеризуется тем, что оно переходит в какое-то состояние, а в него не переходит ни одно состояние, то есть оно не имеет предшественников.

Нашими задачами будут выяснение того, какие состояния $q$ из $Q(b, n, l)$ являются стартовыми и нахождение числа этих состояний. Отметим, что свойство состояния из $Q(b, n, l)$ быть стартовым зависит от параметра $r$, а потому для множества всех стартовых состояний из $Q(b, n, l)$ вводим обозначение $S t(b, r, n, l)$, а для их числа - обозначение $|S t(b, r, n, l)|$.

Для решения этих задач выделим некоторые свойства состояний, эти свойства назовем $s_{v}$-свойствами при $b=r$ и $m_{u}$-свойствами при $b>r$, где $v \in\{1,2,3\}$ и $u \in\{1,2,3,4,5\}$.

Будем говорить, что $q$ из $Q(b, n, l)$ обладает

$s_{1}$-свойством, если при данном $q$ выполнены неравенства $q_{111}>0$ и $q_{112}>0$,

$s_{2}$-свойством, если при данном $q$ существует ресничка с номером $i j k$, где $i \in\{1, \ldots, l\}$, $j \in\left\{1, \ldots, 2^{i-1}\right\}, k \in\{1, \ldots, n\}$, причем $k>1$ при $i=1$ и $k<n$ при $i=l$, такая, что $q_{i j k}=0$ и либо $q_{i j(k-1)}=0$ при $k>1$, либо $q_{(i-1) j^{\prime} n}=0$ при $k=1$ и $i>1$, где ребро $j^{\prime}$ уровня $i-1$ инцидентно ребру $j$ уровня $i$, и при этом имеет место хотя бы одно из следующих условий (ребра $j^{\prime \prime}$ и $j^{\prime \prime \prime}$ уровня $i+1$ инцидентны ребру $j$ уровня $i$ ):

(a) при $k<n-1$ выполнены неравенства $q_{i j(k+1)}>0$ и $q_{i j(k+2)}>0$,

(б) при $k=n-1$ и $i=l$ выполнено неравенство $q_{l j n}>0$,

(в) при $k=n-1$ и $i<l$, если $0<q_{i j n} \leqslant 2^{l-(i+1)} r$, то $q_{(i+1) j^{\prime \prime} 1}>0$ и либо $q_{(i+1) j^{\prime \prime 2}}>0$, либо $q_{(i+1) j^{\prime \prime \prime} 1}>0$; если $2^{l-(i+1)} r<q_{i j n} \leqslant 2^{l-i} r$, то $q_{(i+1) j^{\prime \prime} 1}>0$,

(г) при $k=n$ и $i<l$ выполнены неравенства $q_{(i+1) j^{\prime \prime} 1}>0$ и $q_{(i+1) j^{\prime \prime 2}}>0$;

$s_{3}$-свойством, если $l>1$ и при данном $q$ существует ресничка с номером $i j_{1} 1$, где $i \in\{2,3, \ldots, l\}, j_{1} \in\left\{1, \ldots, 2^{i-1}\right\}$, такая, что $q_{i j_{1} 1}=0$, при этом $q_{i j_{1} 2}>0$, а также имеет место хотя бы одно из следующих условий (ребро $j_{1}$ уровня $i$ инцидентно ребру $j$ уровня $i-1$ и ребру $j_{2}$ уровня $i$ ):

(a) если $q_{(i-1) j n}=1, q_{i j_{2} 1}=0, q_{i j_{2} 2}>0$ и при этом при $n>2$ выполнены неравенства $q_{i j_{1} 3}>0$ и $q_{i j_{2} 3}>0$, и при $n=2$ и $i<l$ для каждого $p$ из $\{1,2\}$ (ребро $j_{p}$ уровня $i$ инцидентно ребрам $j_{p}^{\prime}$ и $j_{p}^{\prime \prime}$ уровня $i+1$ ), если $0<q_{i j_{p} 2} \leqslant 2^{l-(i+1)} r$, то либо $q_{(i+1) j_{p}^{\prime} 1}>0$ и $q_{(i+1) j_{p}^{\prime} 2}>0$, либо $q_{(i+1) j_{p}^{\prime} 1}>0$ и $q_{(i+1) j_{p}^{\prime \prime} 1}>0$, и если $2^{l-(i+1)} r<q_{i j_{p} 2} \leqslant 2^{l-i} r$, то $q_{(i+1) j_{p}^{\prime} 1}>0$,

(б) если $0<q_{(i-1) j n} \leqslant 2^{l-i} r, q_{i j_{2} 1}>0, q_{i j_{2} 2}>0$ и при этом при $n>2$ выполнено неравенство $q_{i j_{1} 3}>0$, а при $n=2$ и $i<l$ (ребро $j_{1}$ уровня $i$ инцидентно ребрам $j_{1}^{\prime}$ и $j_{1}^{\prime \prime}$ уровня $\left.i+1\right)$, если $0<q_{i j_{1} 2} \leqslant 2^{l-(i+1)} r$, то либо $q_{(i+1) j_{1}^{\prime} 1}>0$ и $q_{(i+1) j_{1}^{\prime} 2}>0$, либо $q_{(i+1) j_{1}^{\prime} 1}>0$ и $q_{(i+1) j_{1}^{\prime \prime} 1}>0$, и если $2^{l-(i+1)} r<q_{i j_{1} 2} \leqslant 2^{l-i} r$, то $q_{(i+1) j_{1}^{\prime} 1}>0$, 
(в) если $2^{l-i} r<q_{(i-1) j n} \leqslant 2^{l-(i-1)} r, q_{i j_{2} 1}>0$ и при этом при $n>2$ выполнено неравенство $q_{i j_{1} 3}>0$, а при $n=2$ и $i<l$ (ребро $j_{1}$ уровня $i$ инцидентно ребрам $j_{1}^{\prime}$ и $j_{1}^{\prime \prime}$ уровня $\left.i+1\right)$, если $0<q_{i j_{1} 2} \leqslant 2^{l-(i+1)} r$, то либо $q_{(i+1) j_{1}^{\prime} 1}>0$ и $q_{(i+1) j_{1}^{\prime} 2}>0$, либо $q_{(i+1) j_{1}^{\prime} 1}>0$ и $q_{(i+1) j_{1}^{\prime \prime} 1}>0$, а если $2^{l-(i+1)} r<q_{i j_{1} 2} \leqslant 2^{l-i} r$, то $q_{(i+1) j_{1}^{\prime} 1}>0$.

Чтобы описать $m_{v}$-свойства, $v \in\{1,2,3,4,5\}$, состояния $q$ введем следующее определение.

Три соседние реснички ребра $j$ уровня $i \quad I$-дерева $D^{-1}(b, r, n, l)$ с номерами $k,(k+1)$ и $(k+2)$, где $i \in\{1, \ldots, l\}, j \in\left\{1, \ldots, 2^{i-1}\right\}, k \in\{1, \ldots, n-2\}$, имеющие в состоянии $q$ нагрузки $q_{i j k}, q_{i j(k+1)}$ и $q_{i j(k+2)}$ такие, что

$$
q_{i j k}=2^{l-i} r, \quad 0<q_{i j(k+1)} \leqslant \min \left(2^{l-i} r ; 2^{l-i}(b-r)\right), \quad q_{i j(k+2)}=0,
$$

будем называть $\mu$-тройками.

Распространим это определение на соседние реснички двух инцидентных ребер $j$ и $j^{\prime}$ уровней $i$ и $i+1$, соответственно, где $i \in\{1, \ldots, l-1\}, j \in\left\{1, \ldots, 2^{l-i}\right\}$ и $j^{\prime} \in\left\{1, \ldots, 2^{l-(i+1)}\right\}$, следующим образом. Будем называть $\mu$-тройками также реснички с номерами $i j(n-1), i j n,(i+1) j^{\prime} 1$ или $i j n,(i+1) j^{\prime} 1,(i+1) j^{\prime} 2$, если для состояния $q I$-дерева $D^{-1}(b, r, n, l)$ выполнены соотношения

$$
q_{i j(n-1)}=2^{l-i} r, \quad 0<q_{i j n} \leqslant \min \left(2^{l-i} r, \quad 2^{l-i}(b-r)\right), \quad q_{(i+1) j^{\prime} 1}=0
$$

или соответственно

$$
q_{i j n}=2^{l-i} r, \quad 0<q_{(i+1) j^{\prime} n} \leqslant \min \left(2^{l-(i+1)} r, \quad 2^{l-(i+1)}(b-r)\right), \quad q_{(i+1) j^{\prime} 2}=0 .
$$

Будем говорить, что $q$ из $Q(b, n, l)$ обладает

$m_{1}$-свойством, если выполнено неравенство $q_{111}>2^{l-1}(b-r)$ при данном $q$ в случае $b \geqslant 2 r$, а в случае $r<b<2 r$ выполнено хотя бы одно из условий:

(a) $2^{l-1}(b-r)<q_{111}<2^{l-1} r$ и $q_{112}>0$,

(б) $q_{111}=2^{l-1} r$ и $q_{112}>2^{l-1}(b-r)$,

(в) $q_{111}>2^{l-1} r$;

$m_{2}$-свойством, если при данном $q$ существует цепочка инцидентных ребер, идущая (в направлении к листьям) от ребра $j$ уровня $i$ до ребра $j^{\prime}$ уровня $i^{\prime}$ включительно, где $1 \leqslant i \leqslant i^{\prime} \leqslant l$, такая, что в ребре $j$ уровня $i$ существует ресничка с номером $k$, где $k \in\{1, \ldots, n\}$, такая, что $q_{i j k}=0$ или $q_{i j k}>2^{l-i} r$, и в случае $k<n$ справедливо равенство $q_{i j(k+1)}=0$, а в случае $k=n-$ равенство $q_{(i+1) j^{\prime \prime} 1}=0$, где ребро $j^{\prime \prime}$ уровня $i+1$ принадлежит этой цепочке и инцидентно ребру $j$ уровня $i$, и при этом, начиная с реснички с номером либо $i j(k+2)$ при $k<n-1$, либо $(i+1) j^{\prime \prime} 1$ при $k=n-1$, либо $(i+1) j^{\prime \prime} 2$ при $k=n$, существует $m$, где $m \in\{0,1, \ldots,[(n(l-i+1)-k-2) / 3]\}$, подряд идущих $\mu$-троек с соответствующими ребрам, которым они принадлежат, параметрами и при $\left.i^{\prime}=i+\right](3 m+k+2-n) / n[$ и $k^{\prime}=3 m+k+2-n[(3 m+k+1) / n]$ выполняется хотя бы одно из следующих условий:

(a) $q_{i^{\prime} j^{\prime} k^{\prime}}>2^{l-i^{\prime}} r$, 
(б) $q_{i^{\prime} j^{\prime} k^{\prime}}=2^{l-i^{\prime}} r$ и при $k^{\prime}<n$ справедливо неравенство $q_{i^{\prime} j^{\prime}\left(k^{\prime}+1\right)}>2^{l-i^{\prime}}(b-r)$, и при $k^{\prime}=n$ и $i^{\prime}<l$ справедливо неравенство $q_{\left(i^{\prime}+1\right) j_{1}^{\prime} 1}>2^{l-\left(i^{\prime}+1\right)}(b-r)$, где ребро $j_{1}^{\prime}$ уровня $i^{\prime}+1$ инцидентно ребру $j^{\prime}$ уровня $i^{\prime}$,

(в) $0<q_{i^{\prime} j^{\prime} k^{\prime}}<2^{l-i^{\prime}} r$ и при $k^{\prime}<n$ справедливо неравенство $q_{i^{\prime} j^{\prime}\left(k^{\prime}+1\right)}>0$, а при $k^{\prime}=n$ и $i^{\prime}<l$ выполнено одно из следующих условий (ребра $j_{1}^{\prime}$ и $j_{2}^{\prime}$ уровня $i^{\prime}+1$ инцидентны ребру $j^{\prime}$ уровня $\left.i^{\prime}\right)$ : если $q_{i^{\prime} j^{\prime} k^{\prime}} \neq 2^{l-\left(i^{\prime}+1\right)} r$, то $q_{\left(i^{\prime}+1\right) j_{1}^{\prime} 1}>0$ и $q_{\left(i^{\prime}+1\right) j_{2}^{\prime} 1}>0$, а если $q_{i^{\prime} j^{\prime} k^{\prime}}=2^{l-\left(i^{\prime}+1\right)} r$, то при $b \geqslant 2 r$ справедливо неравенство $q_{\left(i^{\prime}+1\right) j_{1}^{\prime} 1}>2^{l-\left(i^{\prime}+1\right)}(b-r)$, а при $r<b<2 r$ либо $q_{\left(i^{\prime}+1\right) j_{1}^{\prime} 1}>2^{l-\left(i^{\prime}+1\right)}(b-r)$ и $q_{\left(i^{\prime}+1\right) j_{2}^{\prime} 1}>2^{l-\left(i^{\prime}+1\right)}(b-r)$, либо $q_{\left(i^{\prime}+1\right) j_{1}^{\prime} 1}>2^{l-\left(i^{\prime}+1\right)} r$, либо выполняется хотя бы одно из следующих условий: при $q_{\left(i^{\prime}+1\right) j_{1}^{\prime} 1}=2^{l-\left(i^{\prime}+1\right)} r$ справедливо неравенство $q_{\left(i^{\prime}+1\right) j_{1}^{\prime} 2}>2^{l-\left(i^{\prime}+1\right)}(b-r)$, а при $2^{l-\left(i^{\prime}+1\right)}(b-r)<q_{\left(i^{\prime}+1\right) j_{1}^{\prime} 1}<2^{l-\left(i^{\prime}+1\right)} r$ справедливо неравенство $q_{\left(i^{\prime}+1\right) j_{1}^{\prime} 2}>0$;

$m_{3}$-свойством, если $l>1$ и при данном $q$ существует ресничка с номером $i j n$, где $i=1, \ldots, l-1$ и $1 \leqslant j \leqslant 2^{i-1}$, такая, что $q_{i j n}=1$ и при этом $q_{(i+1) j^{\prime} 1}=0$ и $q_{(i+1) j^{\prime \prime} 1}=0$, где ребро $j$ уровня $i$ инцидентно ребрам $j^{\prime}$ и $j^{\prime \prime}$ уровня $i+1$, а также, начиная с ресничек с номерами $(i+1) j^{\prime} 2$ и $(i+1) j^{\prime \prime} 2$, существуют две цепочки из $m$ и $m^{\prime}$, соответственно, где $m, m^{\prime} \in\{0,1,2, \ldots,[(n(l-i)-2) / 3]\}$, подряд идущих (в направлении к листьям) $\mu$-троек с соответствующими ребрам, которым они принадлежат, параметрами до ресничек с номерами $i_{1} j_{1} k_{1}$ и $i_{2} j_{2} k_{2}$ (не включая последние), и таких что при $\left.i_{1}=i+\right](3 m+2) / n\left[, i_{2}=i+\right]\left(3 m^{\prime}+2\right) / n[$, $k_{1}=3 m+2-n[(3 m+1) / n]$ и $k_{2}=3 m^{\prime}+2-n\left[\left(3 m^{\prime}+1\right) / n\right]$ выполнено хотя бы одно из следующих условий:

(а) $q_{i_{1} j_{1} k_{1}}>2^{l-i_{1}} r$ и $q_{i_{2} j_{2} k_{2}}>2^{l-i_{2}} r$,

(б) $q_{i_{1} j_{1} k_{1}}>2^{l-i_{1}} r, q_{i_{2} j_{2} k_{2}}=2^{l-i_{2}} r$ и при $k_{2}<n$ справедливо неравенство $q_{i_{2} j_{2}\left(k_{2}+1\right)}>2^{l-i_{2}}(b-r)$, а при $k_{2}=n$ и $i_{2}<l$ справедливо неравенство $q_{\left(i_{2}+1\right) j_{2}^{\prime} 1}>2^{l-\left(i_{2}+1\right)}(b-r)$, где ребро $j_{2}^{\prime}$ уровня $i_{2}+1$ инцидентно ребру $j_{2}$ уровня $i_{2}$,

(в) $q_{i_{1} j_{1} k_{1}}>2^{l-i_{1}} r, 0<q_{i_{2} j_{2} k_{2}}<2^{l-i_{2}} r$ и при $k_{2}<n$ справедливо неравенство $q_{i_{2} j_{2}\left(k_{2}+1\right)}>0$, а при $k_{2}=n$ и $i_{2}<l$ выполняется одно из следующих условий (ребра $j_{2}^{\prime}$ и $j_{2}^{\prime \prime}$ уровня $i_{2}+1$ инцидентны ребру $j_{2}$ уровня $\left.i_{2}\right)$ : если $q_{i_{2} j_{2} k_{2}} \neq 2^{l-\left(i_{2}+1\right)} r$, то $q_{\left(i_{2}+1\right) j_{2}^{\prime} 1}>0$ и $q_{\left(i_{2}+1\right) j_{2}^{\prime \prime} 1}>0$, если $q_{i_{2} j_{2} k_{2}}=2^{l-\left(i_{2}+1\right)} r$, то справедливо неравенство $q_{\left(i_{2}+1\right) j_{2}^{\prime} 1}>2^{l-\left(i_{2}+1\right)}(b-r)$ при $b \geqslant 2 r$, а при $r<b<2 r$ либо $q_{\left(i_{2}+1\right) j_{2}^{\prime} 1}>2^{l-\left(i_{2}+1\right)}(b-r)$ и $q_{\left(i_{2}+1\right) j_{2}^{\prime \prime} 1}>2^{l-\left(i_{2}+1\right)}(b-r)$, либо $q_{\left(i_{2}+1\right) j_{2}^{\prime} 1}>2^{l-\left(i_{2}+1\right)} r$, либо выполняется хотя бы одно из следующих условий: при $q_{\left(i_{2}+1\right) j_{2}^{\prime} 1}=2^{l-\left(i_{2}+1\right)} r$ верно неравенство $q_{\left(i_{2}+1\right) j_{2}^{\prime} 2}>2^{l-\left(i_{2}+1\right)}(b-r)$, при $2^{l-\left(i_{2}+1\right)}(b-r)<q_{\left(i_{2}+1\right) j_{2}^{\prime} 1}<2^{l-\left(i_{2}+1\right)} r$ справедливо неравенство $q_{\left(i_{2}+1\right) j_{2}^{\prime} 2}>0$,

(г) $q_{i_{1} j_{1} k_{1}}=2^{l-i_{1}} r, q_{i_{2} j_{2} k_{2}}=2^{l-i_{2}} r$ и для каждого $p$ из $\{1,2\}$ при $k_{p}<n$ справедливо неравенство $q_{i_{p} j_{p}\left(k_{p}+1\right)}>2^{l-i_{p}}(b-r)$, а при $k_{p}=n$ и $i_{p}<l-$ неравенство $q_{\left(i_{p}+1\right) j_{p}^{\prime} 1}>2^{l-\left(i_{p}+1\right)}(b-r)$, где ребро $j_{p}^{\prime}$ уровня $i_{p}+1$ инцидентно ребру $j_{p}$ уровня $i_{p}$, 
(д) $0<q_{i_{1} j_{1} k_{1}}<2^{l-i_{1}} r, 0<q_{i_{2} j_{2} k_{2}}<2^{l-i_{2}} r$ и для каждого $p$ из $\{1,2\}$ при $k_{p}<n$ справедливо неравенство $q_{i_{p} j_{p}\left(k_{p}+1\right)}>0$, а при $k_{p}=n$ и $i_{p}<l$ выполняется одно из следующих условий (ребра $j_{p}^{\prime}$ и $j_{p}^{\prime \prime}$ уровня $i_{p}+1$ инцидентны ребру $j_{p}$ уровня $\left.i_{p}\right)$ : если $q_{i_{p} j_{p} k_{p}} \neq 2^{l-\left(i_{p}+1\right)} r$, то $q_{\left(i_{p}+1\right) j_{p}^{\prime} 1}>0$ и $q_{\left(i_{p}+1\right) j_{p}^{\prime \prime} 1}>0$, если $q_{i_{p} j_{p} k_{p}}=2^{l-\left(i_{p}+1\right)} r$, то при $b \geqslant 2 r$ верно неравенство $q_{\left(i_{p}+1\right) j_{p}^{\prime} 1}>2^{l-\left(i_{p}+1\right)}(b-r)$, а при $r<b<2 r$ либо $q_{\left(i_{p}+1\right) j_{p}^{\prime} 1}>2^{l-\left(i_{p}+1\right)}(b-r)$ и $q_{\left(i_{p}+1\right) j_{p}^{\prime \prime} 1}>2^{l-\left(i_{p}+1\right)}(b-r)$, либо $q_{\left(i_{p}+1\right) j_{p}^{\prime} 1}>2^{l-\left(i_{p}+1\right)} r$, либо выполняется хотя бы одно из следующих условий: при $q_{\left(i_{p}+1\right) j_{p}^{\prime} 1}=2^{l-\left(i_{p}+1\right)} r$ верно неравенство $q_{\left(i_{p}+1\right) j_{p}^{\prime} 2}>2^{l-\left(i_{p}+1\right)}(b-r)$, при $2^{l-\left(i_{p}+1\right)}(b-r)<q_{\left(i_{p}+1\right) j_{p}^{\prime} 1}<2^{l-\left(i_{p}+1\right)} r$ верно неравенство $q_{\left(i_{p}+1\right) j_{p}^{\prime} 2}>0$,

(е) $q_{i_{1} j_{1} k_{1}}=2^{l-i_{1}} r, 0<q_{i_{2} j_{2} k_{2}}<2^{l-i_{2}} r$ и при $k_{1}<n$ справедливо неравенство $q_{i_{1} j_{1}\left(k_{1}+1\right)}>2^{l-i_{1}}(b-r)$, при $k_{2}<n-$ неравенство $q_{i_{2} j_{2}\left(k_{2}+1\right)}>0$, при $k_{1}=n$ и $i_{1}<l$ - неравенство $q_{\left(i_{1}+1\right) j_{1}^{\prime} 1}>2^{l-\left(i_{1}+1\right)}(b-r)$, где ребро $j_{1}^{\prime}$ уровня $i_{1}+1$ инцидентно ребру $j_{1}$ уровня $i_{1}$, а при $k_{2}=n$ и $i_{2}<l$ выполняется одно из следующих условий (ребра $j_{2}^{\prime}$ и $j_{2}^{\prime \prime}$ уровня $i_{2}+1$ инцидентны ребру $j_{2}$ уровня $\left.i_{2}\right)$ : если $q_{i_{2} j_{2} k_{2}} \neq 2^{l-\left(i_{2}+1\right)} r$, то $q_{\left(i_{2}+1\right) j_{2}^{\prime} 1}>0$ и $q_{\left(i_{2}+1\right) j_{2}^{\prime \prime} 1}>0$, если $q_{i_{2} j_{2} k_{2}}=2^{l-\left(i_{2}+1\right)} r$, то при $b \geqslant 2 r$ верно неравенство $q_{\left(i_{2}+1\right) j_{2}^{\prime} 1}>2^{l-\left(i_{2}+1\right)}(b-r)$, а при $r<b<2 r$ либо $q_{\left(i_{2}+1\right) j_{2}^{\prime} 1}>2^{l-\left(i_{2}+1\right)}(b-r)$ и $q_{\left(i_{2}+1\right) j_{2}^{\prime \prime} 1}>2^{l-\left(i_{2}+1\right)}(b-r)$, либо $q_{\left(i_{2}+1\right) j_{2}^{\prime} 1}>2^{l-\left(i_{2}+1\right)} r$, либо выполняется хотя бы одно из следующих условий: при $q_{\left(i_{2}+1\right) j_{2}^{\prime} 1}=2^{l-\left(i_{2}+1\right)} r$ верно неравенство $q_{\left(i_{2}+1\right) j_{2}^{\prime} 2}>2^{l-\left(i_{2}+1\right)}(b-r)$, при $2^{l-\left(i_{2}+1\right)}(b-r)<q_{\left(i_{2}+1\right) j_{2}^{\prime} 1}<2^{l-\left(i_{2}+1\right)} r$ справедливо неравенство $q_{\left(i_{2}+1\right) j_{2}^{\prime} 2}>0$;

$m_{4}$-свойством, если $l>1$ и при данном $q$ существует ресничка с номером $i j n$, где $i=1,2,3, \ldots, l-1$ и $1 \leqslant j \leqslant 2^{i-1}$, такая, что $2^{l-(i+1)} r<q_{i j n} \leqslant 2^{l-i} r$ и при этом $q_{(i+1) j^{\prime} 1}=0$ и $q_{(i+1) j^{\prime \prime} 1}>2^{l-(i+1)}(b-r)$, где ребро $j$ уровня $i$ инцидентно ребрам $j^{\prime}$ и $j^{\prime \prime}$ уровня $i+1$, а также, начиная с реснички с номером $(i+1) j^{\prime} 2$, существует цепочка из $m$, где $m \in\{0,1,2, \ldots,[(n(l-i)-2) / 3]\}$, подряд идущих (в направлении к листьям) $\mu$-троек с соответствующими ребрам, которым они принадлежат, параметрами до реснички с номером $i_{1} j_{1} k_{1}$ (не включая последнюю), и такая, что при $\left.i_{1}=i+\right](3 m+2) / n\left[\right.$ и $k_{1}=3 m+2-n[(3 m+1) / n]$ выполнено хотя бы одно из следующих условий:

(a) $q_{i_{1} j_{1} k_{1}}>2^{l-i_{1}} r$

(б) $q_{i_{1} j_{1} k_{1}}=2^{l-i_{1}} r$ и при $k_{1}<n$ верно неравенство $q_{i_{1} j_{1}\left(k_{1}+1\right)}>2^{l-i_{1}}(b-r)$, а при $k_{1}=n$ и $i_{1}<l-$ неравенство $q_{\left(i_{1}+1\right) j_{1}^{\prime} 1}>2^{l-\left(i_{1}+1\right)}(b-r)$, где ребро $j_{1}^{\prime}$ уровня $i_{1}+1$ инцидентно ребру $j_{1}$ уровня $i_{1}$,

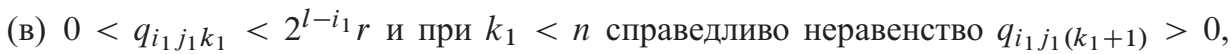
а при $k_{1}=n$ и $i_{1}<l$ выполняется одно из следующих условий (ребра $j_{1}^{\prime}$ и $j_{1}^{\prime \prime}$ уровня $i_{1}+1$ инцидентны ребру $j_{1}$ уровня $\left.i_{1}\right)$ : если $q_{i_{1} j_{1} k_{1}} \neq 2^{l-\left(i_{1}+1\right)} r$, то $q_{\left(i_{1}+1\right) j_{1}^{\prime} 1}>0$ и $q_{\left(i_{1}+1\right) j_{1}^{\prime \prime} 1}>0$, если $q_{i_{1} j_{1} k_{1}}=2^{l-\left(i_{1}+1\right)} r$, то при $b \geqslant 2 r$ справедливо неравенство $q_{\left(i_{1}+1\right) j_{1}^{\prime} 1}>2^{l-\left(i_{1}+1\right)}(b-r)$, а при $r<b<2 r$ либо $q_{\left(i_{1}+1\right) j_{1}^{\prime} 1}>2^{l-\left(i_{1}+1\right)}(b-r)$ и $q_{\left(i_{1}+1\right) j_{1}^{\prime \prime} 1}>2^{l-\left(i_{1}+1\right)}(b-r)$, либо $q_{\left(i_{1}+1\right) j_{1}^{\prime} 1}>2^{l-\left(i_{1}+1\right)} r$, либо выполняется хотя бы одно из следующих условий: 
при $q_{\left(i_{1}+1\right) j_{1}^{\prime} 1}=2^{l-\left(i_{1}+1\right)} r$ верно неравенство $q_{\left(i_{1}+1\right) j_{1}^{\prime} 2}>2^{l-\left(i_{1}+1\right)}(b-r)$, а при $2^{l-\left(i_{1}+1\right)}(b-r)<q_{\left(i_{1}+1\right) j_{1}^{\prime} 1}<2^{l-\left(i_{1}+1\right)} r-$ неравенство $q_{\left(i_{1}+1\right) j_{1}^{\prime} 2}>0$;

$m_{5}$-свойством, если $l>1$ и при данном $q$ существует ресничка с номером $i j n$, где $i=1,2,3, \ldots, l-1$ и $1 \leqslant j \leqslant 2^{i-1}$, такая, что $0<q_{i j n} \leqslant 2^{l-(i+1)} r$ и при этом $q_{(i+1) j^{\prime} 1}=0$ и либо $q_{(i+1) j^{\prime \prime} 1}>2^{l-(i+1)} r$, либо $0<q_{(i+1) j^{\prime \prime} 1}<2^{l-(i+1)} r$ и $q_{(i+1) j^{\prime \prime} 2}>0$, либо $q_{(i+1) j^{\prime \prime} 1}=2^{l-(i+1)} r$ и $q_{(i+1) j^{\prime \prime} 2}>2^{l-(i+1)}(b-r)$, где ребро $j$ уровня $i$ инцидентно ребрам $j^{\prime}$ и $j^{\prime \prime}$ уровня $i+1$, а также, начиная с реснички с номером $(i+1) j^{\prime} 2$, существует цепочка из $m$, где $m \in\{0,1,2, \ldots,[(n(l-i)-2) / 3]\}$, подряд идущих (в направлении к листьям) $\mu$-троек с соответствующими ребрам, которым они принадлежат, параметрами до реснички с номером $i_{1} j_{1} k_{1}$ (не включая последнюю), и такая, что при $\left.i_{1}=i+\right](3 m+2) / n\left[\right.$ и $k_{1}=3 m+2-n[(3 m+1) / n]$ выполнено хотя бы одно из следующих условий:

(a) $q_{i_{1} j_{1} k_{1}}>2^{l-i_{1}} r$,

(б) $q_{i_{1} j_{1} k_{1}}=2^{l-i_{1}} r$ и при $k_{1}<n$ верно неравенство $q_{i_{1} j_{1}\left(k_{1}+1\right)}>2^{l-i_{1}}(b-r)$, а при $k_{1}=n$ и $i_{1}<l$ справедливо неравенство $q_{\left(i_{1}+1\right) j_{1}^{\prime} 1}>2^{l-\left(i_{1}+1\right)}(b-r)$, где ребро $j_{1}^{\prime}$ уровня $i_{1}+1$ инцидентно ребру $j_{1}$ уровня $i_{1}$,

(в) $0<q_{i_{1} j_{1} k_{1}}<2^{l-i_{1}} r$ и при $k_{1}<n$ справедливо неравенство $q_{i_{1} j_{1}\left(k_{1}+1\right)}>0$, а при $k_{1}=n$ и $i_{1}<l$ выполняется одно из следующих условий (ребра $j_{1}^{\prime}$ и $j_{1}^{\prime \prime}$ уровня $i_{1}+1$ инцидентны ребру $j_{1}$ уровня $\left.i_{1}\right)$ : если $q_{i_{1} j_{1} k_{1}} \neq 2^{l-\left(i_{1}+1\right)} r$, то $q_{\left(i_{1}+1\right) j_{1}^{\prime} 1}>0$ и $q_{\left(i_{1}+1\right) j_{1}^{\prime \prime} 1}>0$, если $q_{i_{1} j_{1} k_{1}}=2^{l-\left(i_{1}+1\right)} r$, то $q_{\left(i_{1}+1\right) j_{1}^{\prime} 1}>2^{l-\left(i_{1}+1\right)}(b-r)$ при $b \geqslant 2 r$, а при $r<b<2 r$ либо $q_{\left(i_{1}+1\right) j_{1}^{\prime} 1}>2^{l-\left(i_{1}+1 b-r\right)}$ и $q_{\left(i_{1}+1\right) j_{1}^{\prime \prime} 1}>2^{l-\left(i_{1}+1\right)}(b-r)$, либо

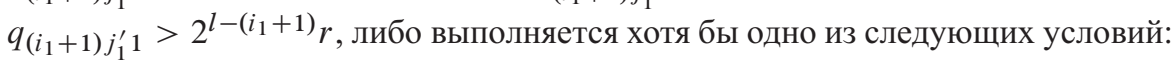
при $q_{\left(i_{1}+1\right) j_{1}^{\prime} 1}=2^{l-\left(i_{1}+1\right)} r$ верно неравенство $q_{\left(i_{1}+1\right) j_{1}^{\prime} 2}>2^{l-\left(i_{1}+1\right)}(b-r)$, а при $2^{l-\left(i_{1}+1\right)}(b-r)<q_{\left(i_{1}+1\right) j_{1}^{\prime} 1}<2^{l-\left(i_{1}+1\right)} r-$ неравенство $q_{\left(i_{1}+1\right) j_{1}^{\prime} 2}>0$.

Пусть

$$
\mathbf{N}_{k}=\{1,2, \ldots, k\}, \quad S=\left\{s_{v} \mid v \in \mathbf{N}_{3}\right\}, \quad M=\left\{m_{v} \mid v \in \mathbf{N}_{5}\right\}
$$

Класс всех состояний $q$ из $Q(b, n, l)$ с $s_{v}$-свойством при $s_{v} \in S$ обозначим через $K_{s_{v}}$, а класс всех состояний $q$ из $Q(b, n, l)$ с $m_{v}$-свойством при $m_{v} \in M$ обозначим через $K_{m_{v}}$.

Справедливы следующие утверждения.

Теорема 2. При $b=r$

$$
t(b, r, n, l)= \begin{cases}K_{s_{1}}, & \text { если } l=1, n=2, \\ K_{s_{1}} \cup K_{s_{2}}, & \text { если } l=1, n>2, \\ K_{s_{1}} \cup K_{s_{2}} \cup K_{s_{3}}, & \text { если } l \geqslant 2, n \geqslant 2,\end{cases}
$$

где $K_{s_{v}} \neq \varnothing$ при всех $v$ из $\mathbf{N}_{3}$, и если $j \in\{(2),(3)\}$, то для элементов строки (j) при $v \neq$ и справедливо соотношение $K_{s_{v}} \nsubseteq K_{s_{u}}$.

Теорема 3. При $b>r$

$$
t(b, r, n, l)= \begin{cases}K_{m_{1}}, & \text { если } l=1 \text { и } n=2, \\ K_{m_{1}} \cup K_{m_{2}}, & \text { если } l=1 \text { и } n>2, \\ \bigcup_{v \in \mathbf{N}_{5}} K_{m_{v}}, & \text { если } l \geqslant 2 \text { и } n \geqslant 2,\end{cases}
$$


где $K_{m_{v}} \neq \varnothing$ при всех $v$ из $\mathbf{N}_{5}$, и если $j \in\{(2),(3)\}$, то для элементов строки ( $\left.j\right)$ при $v \neq u$ справедливо соотночение $K_{m_{v}} \nsubseteq K_{m_{u}}$.

Теорема 4. Справедливы неравенства

$$
C \leqslant \frac{|S t(b, r, n, l)|}{|Q(b, n, l)|} \leqslant 1,
$$

2de

$$
C=\left(\frac{2^{l-1} b}{2^{l-1} b+1}\right)^{2}
$$

nрu $b=r$,

$$
C=\frac{\left(2^{l-1}\right)^{2} r b}{\left(2^{l-1} b+1\right)^{2}}
$$

nрu $r<b<2 r u$

$$
C=\frac{2^{l-1} r}{2^{l-1} b+1}
$$

при $b \geqslant 2 r$.

Следствие 3. При $l \rightarrow \infty$

(a) $|\operatorname{St}(b, r, n, l)| \sim|Q(b, n, l)|$, если $b=r$,

(б) $|S t(b, r, n, l)| \asymp|Q(b, n, l)|$, если $b>r$.

Его справедливость вытекает из теоремы 4 с учетом того, что при $l \rightarrow \infty$, если $b=r$, то $C \rightarrow 1$, а если $b>r$, то $C \rightarrow r / b$.

\section{3. Доказательства теорем}

Докажем ряд вспомогательных утверждений.

Лемма 1. При $b=r, l=1, n=2$ состояние $q \in Q(b, n, l) I$-дерева $D^{-1}(b, r, n, l)$ является стартовым тогда и только тогда, когда $q \in K_{s_{1}}$.

Доказательство. Нетрудно видеть, что если $q$ из $Q(b, n, l)$ принадлежит классу $K_{s_{1}}$, то $q$ является стартовым. Покажем, что, если $q$ является стартовым, то оно принадлежит $K_{s_{1}}$. Рассмотри все возможные случаи для нагрузок ресничек $I$-дерева $D^{-1}(b, r, n, l)$ в состоянии $q$ :

(1) $q_{111}=0$ и $q_{112}=0$;

(2) $q_{111}=0$ и $q_{112}>0$;

(3) $q_{111}>0$ и $q_{112}=0$;

(4) $q_{111}>0$ и $q_{112}>0$.

В случаях (1)-(3) состояние $q$ не является стартовым. Действительно, в каждом из этих случаев существует предшественник $q^{\prime}$ состояния $q$ такой, что в случае (1) $q_{111}^{\prime}=1$ и $q_{112}^{\prime}=0$, в случае (2) $q_{111}^{\prime}=1$ и $q_{112}^{\prime}=q_{112}$, в случае (3) $q_{111}^{\prime}=0$ и $q_{112}^{\prime}=q_{111}$.

В случае (4) состояние $q$, очевидно, является стартовым и при этом обладает $s_{1}$-свойством. Следовательно, $q \in K_{s_{1}}$. Лемма доказана. 
Лемма 2. Если $b=r, l=1$ и $n>2$, то справедливы следующие утверждения:

(а) состояние $q \in Q(b, n, l) I$-дерева $D^{-1}(b, r, n, l)$ является стартовым тогда и только тогда, когда $q \in K_{s_{v}}$ при некотором $v \in \mathbf{N}_{2}$,

(б) $K_{s_{u}} \nsubseteq K_{s_{v}}$ для любых $s_{u}, s_{v} \in\left\{s_{1}, s_{2}\right\}$ при $u \neq v$.

Доказательство. Докажем утверждение (а). Легко видеть, что, если $q$ из $Q(b, n, l)$ принадлежит классу $K_{s_{v}}$, где $v \in \mathbf{N}_{2}$, то $q$ является стартовым.

Покажем, что, если $q$ является стартовым, то оно принадлежит хотя бы одному из классов $K_{s_{v}}$, где $v \in \mathbf{N}_{2}$. Заметим, что если $q$ стартовое, то для него выполняется хотя бы одно из следующих условий:

(1) $q_{111}>0$ и $q_{112}>0$;

(2) $q_{11 n}>0, q_{11(n-1)}=0$ и $q_{11(n-2)}=0$;

(3) при $n>3$ существуют четыре соседние реснички с номерами $11 k, 11(k+1)$, $11(k+2)$ и $11(k+3)$, где $k<n-2$, такие, что $q_{11 k}=0, q_{11(k+1)}=0, q_{11(k+2)}>0$ и $q_{11(k+3)}>0$.

Действительно, пусть для $q$ не выполняется ни одно из условий (1), (2), (3). Тогда существует предшественник $q^{\prime}$ состояния $q$ такой, что для всех пар ресничек с номерами 11( $p-1)$ и $11 p, p>1$ таких, что $q_{11(p-1)}>0$ и $q_{11 p}=0$, справедливы равенства $q_{11(p-1)}^{\prime}=0$ и $q_{11 p}^{\prime}=q_{11(p-1)}$, а при $q_{111}=0$ равенство $q_{111}^{\prime}=1$, остальные реснички $I$-дерева $D^{-1}(b, r, n, l)$ в состоянии $q^{\prime}$ имеют те же нагрузки, что и в состоянии $q$. Следовательно, при невыполнении ни одного из трех указанных условий состояние $q$ стартовым не является, а при выполнении хотя бы одного из этих условий $q$, очевидно, является стартовым.

Если для $q$ выполняется условие (1), то $q$ обладает $s_{1}$-свойством, а следовательно, $q \in K_{s_{1}}$. Если для $q$ выполняется условие (2) или (3), то $q$ обладает $s_{2}$-свойством, а следовательно, $q \in K_{s_{2}}$.

Таким образом, показано, что если $q$ является стартовым, то $q \in K_{s_{v}}$, где $v \in \mathbf{N}_{2}$.

Докажем утверждение (б). Пусть состояние $q^{1}$ из $K_{s_{1}}$ такое, что $q_{111}^{1}=1, q_{112}^{1}=1$, а остальные реснички в $I$-дереве $D^{-1}(b, r, n, l)$ имеют нулевые нагрузки. Легко видеть, что $q^{1} \notin K_{s_{2}}$. Следовательно, $K_{s_{1}} \nsubseteq K_{s_{2}}$.

Пусть состояние $q^{2}$ из $K_{S_{2}}$ такое, что $q_{11 n}^{2}=1$, а остальные реснички в $I$-дереве $D^{-1}(b, r, n, l)$ имеют нулевые нагрузки. Легко видеть, что $q^{2} \notin K_{s_{1}}$. Следовательно, $K_{s_{2}} \nsubseteq K_{s_{1}}$. Лемма доказана.

Лемма 3. Если $b=r, l \geqslant 2 u n \geqslant 2$, то справедливы следующче утверждения:

(а) состояние $q$ из $Q(b, n, l) I$-дерева $D^{-1}(b, r, n, l)$ является стартовым тогда и только тогда, когда $q \in K_{s_{v}}$ при некотором $v$ таком, что $v \in \mathbf{N}_{3}$,

(б) $K_{s_{u}} \not K_{s_{v}}$ для любых $s_{u}, s_{v}$ из $S$ при $u \neq v$.

Доказательство. Докажем утверждение (а). Нетрудно видеть, что если $q$ из $Q(b, n, l)$ принадлежит классу $K_{s_{v}}$, где $v \in \mathbf{N}_{3}$, то $q$ является стартовым.

Покажем, что, если $q$ является стартовым, то оно обладает хотя бы одним из $s_{v}$-свойств, а следовательно, принадлежит классу $K_{s_{v}}$, где $v \in \mathbf{N}_{3}$. Предположим, что состояние $q$ является стартовым и при этом не обладает ни одним из указанных трех свойств. Тогда 
у $q$ существует предшественник $q^{\prime}$, который можно построить следующим образом. Для каждого ребра $j$ уровня $i$ для всех пар ресничек с номерами $i j(k-1)$ и $i j k, k>1$ таких, что $q_{i j(k-1)}>0$ и $q_{i j k}=0$, справедливы равенства $q_{i j(k-1)}^{\prime}=0$ и $q_{i j k}^{\prime}=q_{11(k-1)}$, где $i \in \mathbf{N}_{l}$ и $j \in \mathbf{N}_{2^{i-1}}$, а при $q_{111}=0$ равенство $q_{111}^{\prime}=1$. Для каждой тройки инцидентных ребер, состоящей из ребра $j$ уровня $i$ и ребер $j^{\prime}$ и $j^{\prime \prime}$ уровня $i+1$, где $i \in \mathbf{N}_{l-1}$ и $j \in \mathbf{N}_{2^{i-1}}$ такой, что

(1) $1<q_{i j n} \leqslant 2^{l-(i+1)} r, q_{(i+1) j^{\prime} 1}=0$ и $q_{(i+1) j^{\prime \prime} 1}=0$ и справедливы равенства $q_{i j n}^{\prime}=0, q_{(i+1) j^{\prime} 1}^{\prime}=1$ и $q_{(i+1) j^{\prime \prime} 1}^{\prime}=q_{i j n}-1$;

(2) $0<q_{i j n} \leqslant 2^{l-(i+1)} r, q_{(i+1) j^{\prime} 1}=0$ и $q_{(i+1) j^{\prime} 2}>0$, и соотношения $q_{i j n}^{\prime}=0$, $q_{(i+1) j^{\prime} 1}^{\prime}=q_{i j n}$ и $q_{(i+1) j^{\prime} 2}^{\prime}=q_{(i+1) j^{\prime} 2}$ справедливы тогда и только тогда, когда выполняется одно из следующих условий (где ребра $j_{1}^{\prime}$ и $j_{2}^{\prime}$ уровня $i+2$ инцидентны ребру $j^{\prime}$ уровня $\left.i+1\right)$ :

$$
\begin{aligned}
- & n>2 \text { и } q_{(i+1) j^{\prime} 3}>0, \\
- & n=2 \text { и } i=l-1, \\
- & n=2, i<l-1, q_{(i+1) j^{\prime} 2} \leqslant 2^{l-(i+2)} r, q_{(i+2) j_{1}^{\prime} 1}>0 \text { и } q_{(i+2) j_{1}^{\prime} 2}>0 \text { или } \\
& q_{(i+2) j_{2}^{\prime} 1>0,} \\
- & n=2, i<l-1, q_{(i+1) j^{\prime} 2}>2^{l-(i+2)} r \text { и } q_{(i+2) j_{1}^{\prime} 1}>0 ;
\end{aligned}
$$

(3) $2^{l-(i+1)} r<q_{i j n} \leqslant 2^{l-i} r, q_{(i+1) j^{\prime} 1}=0$ и $q_{(i+1) j^{\prime \prime} 1}=0$, справедливы равенства $q_{i j n}^{\prime}=0, q_{(i+1) j^{\prime} 1}^{\prime}=2^{l-(i+1)} r$ и $q_{(i+1) j^{\prime \prime} 1}^{\prime}=q_{i j n}-2^{l-(i+1)} r$.

Остальные реснички $I$-дерева $D^{-1}(b, r, n, l)$ в состоянии $q^{\prime}$ имеют те же нагрузки, что и в состоянии $q$.

Получено противоречие, так как стартовое состояние не имеет предшественников.

Таким образом, показано, что если $q$ является стартовым, то $q \in K_{s_{v}}$, где $v \in \mathbf{N}_{3}$.

Докажем утверждение (б). Пусть состояние $q^{1}$ из $K_{s_{1}}$ такое, что $q_{111}^{1}=1$ и $q_{112}^{1}=1$, а нагрузки остальных ресничек в $I$-дереве $D^{-1}(b, r, n, l)$ равны нулю. Легко видеть, что $q^{1} \notin K_{s_{2}} \cup K_{s_{3}}$. Следовательно, $K_{s_{1}} \not K_{s_{v}}$ при всех $v \in\{2,3\}$.

Пусть состояние $q^{2}$ из $K_{S_{2}}$ такое, что $q_{11 n}=1$, а нагрузки остальных ресничек в $I$-дереве $D^{-1}(b, r, n, l)$ равны нулю. Легко видеть, что $q^{2} \notin K_{s_{1}} \cup K_{s_{3}}$. Следовательно, $K_{s_{2}} \nsubseteq K_{s_{v}}$ при всех $v \in\{1,3\}$.

Пусть состояние $q^{3}$ из $K_{s_{3}}$ такое, что $q_{(l-1) 1 n}^{3}=1, q_{l 12}^{3}=1, q_{l 22}^{3}=1$ и если $n>2$, то еще $q_{l 13}^{3}=1$ и $q_{l 23}^{3}=1$, а нагрузки остальных ресничек в $I$-дереве $D^{-1}(b, r, n, l)$ равны нулю. Легко видеть, что $q^{3} \notin K_{s_{1}} \cup K_{s_{2}}$. Следовательно, $K_{s_{3}} \nsubseteq K_{s_{v}}$ при всех $v \in\{1,2\}$. Лемма доказана.

Лемма 4. При $b>r, l=1 u n=2$, состояние q из $Q(b, n, l) I$-дерева $D^{-1}(b, r, n, l)$ является стартовым тогда и только тогда, когда $q \in K_{m_{1}}$.

Доказательство. Нетрудно видеть, что если $q$ из $Q(b, n, l)$ принадлежит классу $K_{m_{1}}$, то $q$ является стартовым.

Покажем, что если $q$ является стартовым, то оно принадлежит $K_{m_{1}}$. Рассмотрим все возможные случаи для нагрузок ресничек $I$-дерева $D^{-1}(b, r, n, l)$ в состоянии $q$, это

(1) $q_{111}>b-r$ и $q_{112} \geqslant 0$;

(2) $q_{111} \leqslant b-r$ и $q_{112} \geqslant 0$. 
Состояние $q$ в случае (2) не является стартовым, так как существует предшественник $q^{\prime}$ состояния $q$ такой, что $q_{111}^{\prime}=q_{111}+r$ и $q_{112}^{\prime}=q_{112}$.

Если $b \geqslant 2 r$, то состояние $q$ в случае (1), очевидно, является стартовым и при этом обладает $m_{1}$-свойством, а следовательно, $q \in K_{m_{1}}$.

Если $r<b<2 r$, то рассмотрим все возможные варианты нагрузок ресничек $I$-дерева в состоянии $q$ в случае (1), это

(a) $q_{111}>r$ и $q_{112} \geqslant 0$;

(б) $q_{111}=r$ и $q_{112}>b-r$;

(в) $q_{111}=r$ и $q_{112} \leqslant b-r$;

(г) $b-r<q_{111}<r$ и $q_{112}>0$;

(д) $b-r<q_{111}<r$ и $q_{112}=0$.

В случаях (в) и (д) состояние $q$ не является стартовым, так как в каждом из этих случаев существует предшественник $q^{\prime}$ состояния $q$ такой, что в случае (в) $q_{111}^{\prime}=0$ и $q_{112}^{\prime}=q_{112}+r$, а в случае (д) $q_{111}^{\prime}=0$ и $q_{112}^{\prime}=q_{111}$.

Легко видеть, что в случаях (a), (б) и (г) состояние $q$ является стартовым и при этом обладает $m_{1}$-свойством, а следовательно, $q \in K_{m_{1}}$. Лемма доказана.

Лемма 5. Если $b>r, l=1$ u $n>2$, то справедливы следующие утверждения:

(а) состояние q из $Q(b, n, l) I$-дерева $D^{-1}(b, r, n, l)$ является стартовым тогда и только тогда, когда $q \in K_{m_{v}}$ при некотором $v$ таком, что $v \in \mathbf{N}_{2}$,

(б) $K_{m_{u}} \nsubseteq K_{m_{v}}$ для любых $m_{u}, m_{v}$ из $\left\{m_{1}, m_{2}\right\}$ при $u \neq v$.

Доказательство. Докажем утверждение (а). Нетрудно видеть, что, если $q \in Q(b, n, l)$ принадлежит классу $K_{m_{v}}$, где $v \in \mathbf{N}_{2}$, то $q$ является стартовым.

Покажем, что если $q$ является стартовым, то оно обладает хотя бы одним из $m_{v}$-свойств, а следовательно, принадлежит классу $K_{m_{v}}$, где $v \in \mathbf{N}_{2}$. Предположим, что состояние $q$ является стартовым и при этом не обладает ни одним из указанных двух свойств. Тогда у $q$ существует предшественник $q^{\prime}$, который можно построить следующим образом. Для всех пар ресничек с номерами 11( $k-1)$ и $11 k, k>1$ таких, что $0<q_{11(k-1)} \leqslant r$ и $q_{11 k}=0$, справедливы равенства $q_{11(k-1)}^{\prime}=0$ и $q_{11 k}^{\prime}=q_{11(k-1)}$. Если $b \geqslant 2 r$, то при $0 \leqslant q_{111} \leqslant r$ и $q_{112}>0$ или при $r<q_{111} \leqslant b-r$ справедливо равенство $q_{111}^{\prime}=q_{111}+r$. Если $b<2 r$, то при $0 \leqslant q_{111} \leqslant b-r$ и $q_{112}>0$ справедливо равенство $q_{111}^{\prime}=q_{111}+r$, а при $q_{111}=r$ и $q_{112}>0$ справедливы равенства $q_{111}^{\prime}=0$ и $q_{112}^{\prime}=q_{112}+r$. Остальные реснички $I$-дерева $D^{-1}(b, r, n, l)$ в состоянии $q^{\prime}$ имеют те же нагрузки, что и в состоянии $q$.

Получили противоречие, так как стартовое состояние не имеет предшественников. Таким образом, показано, что если $q$ является стартовым, то $q \in K_{m_{v}}$, где $v \in \mathbf{N}_{2}$.

Докажем утверждение (б). Пусть состояние $q^{1}$ из $K_{m_{1}}$ такое, что $q_{111}^{1}=b$, а остальные реснички в $I$-дереве $D^{-1}(b, r, n, l)$ имеют нулевые нагрузки. Легко видеть, что $q^{1} \notin K_{m_{2}}$. Следовательно, $K_{m_{1}} \nsubseteq K_{m_{2}}$.

Пусть состояние $q^{2}$ из $K_{m_{2}}$ такое, что $q_{11 n}^{2}=1$, а остальные реснички в $I$-дереве $D^{-1}(b, r, n, l)$ имеют нулевые нагрузки. Легко видеть, что $q^{2} \notin K_{m_{1}}$. Следовательно, $K_{m_{2}} \not K_{m_{1}}$. Лемма доказана.

Лемма 6. Если $b>r, l \geqslant 2 u n \geqslant 2$, то справедливы следующие утверждения: 
(a) состояние $q$ из $Q(b, n, l) I$-дерева $D^{-1}(b, r, n, l)$ является стартовым тогда и только тогда, когда $q \in K_{m_{v}}$ при некотором $v$ таком, что $v \in \mathbf{N}_{5}$;

(б) $K_{m_{u}} \nsubseteq K_{m_{v}}$ для любых $m_{u}, m_{v}$ из $М$ при $u \neq v$.

Доказательство. Докажем утверждение (а). Нетрудно видеть, что если $q$ из $Q(b, n, l)$ принадлежит классу $K_{m_{v}}$, где $v \in \mathbf{N}_{5}$, то $q$ является стартовым.

Покажем, что, если $q$ является стартовым, то оно обладает хотя бы одним из $m_{v}$-свойств, а следовательно, принадлежит классу $K_{m_{v}}$, где $v \in \mathbf{N}_{5}$. Предположим, что состояние $q$ является стартовым и при этом не обладает ни одним из указанных пяти свойств. Тогда у $q$ существует предшественник $q^{\prime}$, который можно построить следующим образом. Для каждого ребра $j$ уровня $i$, где $i \in \mathbf{N}_{l}$ и $j \in \mathbf{N}_{2^{i-1}}$, для всех пар ресничек с номерами $i j(k-1)$ и $i j k, k>1$, таких, что $0<q_{i j(k-1)} \leqslant 2^{l-i} r$ и $q_{i j k}=0$, справедливы равенства $q_{i j(k-1)}^{\prime}=0$ и $q_{i j k}^{\prime}=q_{i j(k-1)}$. Если $b \geqslant 2 r$, то при $0 \leqslant q_{111} \leqslant 2^{l-1} r$ и $q_{112}>0$ или при $2^{l-1} r<q_{111} \leqslant 2^{l-1}(b-r)$ справедливо равенство $q_{111}^{\prime}=q_{111}+2^{l-1} r$. Если $b<2 r$, то при $0 \leqslant q_{111} \leqslant 2^{l-1}(b-r)$ и $q_{112}>0$ справедливо равенство $q_{111}^{\prime}=q_{111}+2^{l-1} r$, а при $q_{111}=2^{l-1} r$ и $q_{112}>0-$ равенства $q_{111}^{\prime}=0$ и $q_{112}^{\prime}=q_{112}+2^{l-1} r$.

Для каждой тройки инцидентных ребер, состоящей из ребра $j$ уровня $i$ и ребер $j^{\prime}$ и $j^{\prime \prime}$ уровня $i+1$, где $i \in \mathbf{N}_{l-1}$ и $j \in \mathbf{N}_{2^{i-1}}$, такой, что

(1) $1<q_{i j n} \leqslant 2^{l-(i+1)} r, q_{(i+1) j^{\prime} 1}=0$ и $q_{(i+1) j^{\prime \prime} 1}=0$, верны равенства $q_{i j n}^{\prime}=0$, $q_{(i+1) j^{\prime} 1}^{\prime}=1$ и $q_{(i+1) j^{\prime \prime} 1}^{\prime}=q_{i j n}-1$;

(2) $0<q_{i j n} \leqslant 2^{l-(i+1)} r$ и $q_{(i+1) j^{\prime} 1}=0$, верны равенства $q_{i j n}^{\prime}=0$ и $q_{(i+1) j^{\prime} 1}^{\prime}=q_{i j n}$ тогда и только тогда, когда, начиная с реснички с номером $(i+1) j^{\prime} 2$, существует цепочка из $m$, где $m \in\{0,1, \ldots,[(n(l-i)-2) / 3]\}$, подряд идущих (в направлении к листьям) $\mu$-троек с соответствующими ребрам, которым они принадлежат, параметрами до реснички с номером $i_{1} j_{1} k_{1}$ (не включая последнюю), и такая, что при $\left.i_{1}=i+\right](3 m+2) / n\left[\right.$ и $k_{1}=3 m+2-n[(3 m+1) / n]$ выполняется хотя бы одно из следующих условий:

(a) $q_{i_{1} j_{1} k_{1}}>2^{l-i_{1}} r$,

(б) $q_{i_{1} j_{1} k_{1}}=2^{l-i_{1}} r$ и при $k_{1}<n$ верно неравенство $q_{i_{1} j_{1}\left(k_{1}+1\right)}>2^{l-i_{1}}(b-r)$, а при $k_{1}=n$ и $i_{1}<l$ выполнено неравенство $q_{\left(i_{1}+1\right) j_{1}^{\prime} 1}>2^{l-\left(i_{1}+1\right)}(b-r)$, где ребро $j_{1}^{\prime}$ уровня $i_{1}+1$ инцидентно ребру $j_{1}$ уровня $i_{1}$,

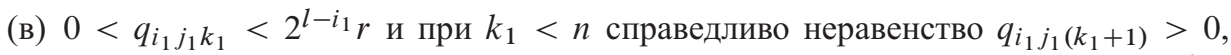
а при $k_{1}=n$ и $i_{1}<l$ выполняется одно из следующих условий (ребра $j_{1}^{\prime}$ и $j_{1}^{\prime \prime}$ уровня $i_{1}+1$ инцидентны ребру $j_{1}$ уровня $\left.i_{1}\right)$ : если $q_{i_{1} j_{1} k_{1}} \neq 2^{l-\left(i_{1}+1\right)} r$, то $q_{\left(i_{1}+1\right) j_{1}^{\prime} 1}>0$ и $q_{\left(i_{1}+1\right) j_{1}^{\prime \prime} 1}>0$, а если $q_{i_{1} j_{1} k_{1}}=2^{l-\left(i_{1}+1\right)} r$, то при $b \geqslant 2 r$ справедливо неравенство $q_{\left(i_{1}+1\right) j_{1}^{\prime} 1}>2^{l-\left(i_{1}+1\right)}(b-r)$, а при $r<b<2 r$ либо $q_{\left(i_{1}+1\right) j_{1}^{\prime} 1}>2^{l-\left(i_{1}+1\right)}(b-r)$ и $q_{\left(i_{1}+1\right) j_{1}^{\prime \prime} 1}>2^{l-\left(i_{1}+1\right)}(b-r)$, либо $q_{\left(i_{1}+1\right) j_{1}^{\prime} 1}>2^{l-\left(i_{1}+1\right)} r$, либо выполняется хотя бы одно из следующих условий: при $q_{\left(i_{1}+1\right) j_{1}^{\prime} 1}=2^{l-\left(i_{1}+1\right)} r$ верно неравенство $q_{\left(i_{1}+1\right) j_{1}^{\prime} 2}>2^{l-\left(i_{1}+1\right)}(b-r)$, а при $2^{l-\left(i_{1}+1\right)}(b-r)<q_{\left(i_{1}+1\right) j_{1}^{\prime} 1}<2^{l-\left(i_{1}+1\right)} r-$ неравенство $q_{\left(i_{1}+1\right) j_{1}^{\prime} 2}>0$;

(3) $2^{l-(i+1)} r<q_{i j n} \leqslant 2^{l-i} r, q_{(i+1) j^{\prime} 1}=0$ и $0 \leqslant q_{(i+1) j^{\prime \prime} 1} \leqslant 2^{l-(i+1)}(b-r)$, справедливы равенства $q_{i j n}^{\prime}=0, q_{(i+1) j^{\prime} 1}^{\prime}=q_{i j n}-2^{l-(i+1)} r$ и $q_{(i+1) j^{\prime \prime} 1}^{\prime}=q_{(i+1) j^{\prime \prime} 1}+2^{l-(i+1)} r$. 
Остальные реснички $I$-дерева $D^{-1}(b, r, n, l)$ в состоянии $q^{\prime}$ имеют те же нагрузки, что и в состоянии $q$.

Получили противоречие, так как стартовое состояние не имеет предшественников.

Таким образом, показано, что если $q$ является стартовым, то $q \in K_{m_{v}}$, где $v \in \mathbf{N}_{5}$.

Докажем утверждение (б). Пусть состояние $q^{1}$ из $K_{m_{1}}$ такое, что $q_{111}^{1}=2^{l-1} b$, а нагрузки остальных ресничек в $I$-дереве $D^{-1}(b, r, n, l)$ равны нулю. Легко видеть, что $q^{1} \notin \bigcup_{v \in \mathbf{N}_{5} \backslash\{1\}} K_{m_{v}}$. Следовательно, $K_{m_{1}} \not K_{m_{v}}$ при всех $v \in \mathbf{N}_{5} \backslash\{1\}$.

Пусть состояние $q^{2}$ из $K_{m_{2}}$ такое, что $q_{11 n}=1$, а нагрузки остальных ресничек в $I$-дереве $D^{-1}(b, r, n, l)$ равны нулю. Легко видеть, что $q^{2} \notin \bigcup_{v \in \mathbf{N}_{5} \backslash\{2\}} K_{m_{v}}$. Следовательно, $K_{m_{2}} \nsubseteq K_{m_{v}}$ при всех $v \in \mathbf{N}_{5} \backslash\{2\}$.

Пусть состояние $q^{3}$ из $K_{m_{3}}$ такое, что $q_{(l-1) 1 n}^{3}=1, q_{l 12}^{3}=b, q_{l 22}^{3}=b$, а нагрузки остальных ресничек в $I$-дереве $D^{-1}(b, r, n, l)$ равны нулю. Легко видеть, что $q^{3} \notin \bigcup_{v \in \mathbf{N}_{5} \backslash\{3\}} K_{m_{v}}$. Следовательно, $K_{m_{3}} \not K_{m_{v}}$ при всех $v \in \mathbf{N}_{5} \backslash\{3\}$.

Пусть состояние $q^{4}$ из $K_{m_{4}}$ такое, что $q_{(l-1) 1(n-1)}^{4}=1, q_{(l-1) 1 n}^{4}=2 r, q_{l 12}^{4}=b$, $q_{l 21}^{4}=b$, а нагрузки остальных ресничек в $I$-дереве $D^{-1}(b, r, n, l)$ равны нулю. Легко видеть, что $q^{4} \notin \bigcup_{v \in \mathbf{N}_{5} \backslash\{4\}} K_{m_{v}}$. Следовательно, $K_{m_{4}} \not K_{m_{v}}$ при всех $v \in \mathbf{N}_{5} \backslash\{4\}$.

Пусть состояние $q^{5}$ из $K_{m_{5}}$ такое, что $q_{(l-1) 1(n-1)}^{5}=1, q_{(l-1) 1 n}^{5}=r, q_{l 12}^{5}=b, q_{l 21}^{5}=b$, а нагрузки остальных ресничек в $I$-дереве $D^{-1}(b, r, n, l)$ равны нулю. Легко видеть, что $q^{5} \notin \bigcup_{v \in \mathbf{N}_{4}} K_{m_{v}}$. Следовательно, $K_{m_{5}} \not \subset K_{m_{v}}$ при всех $v \in \mathbf{N}_{4}$. Лемма доказана.

Доказательство теоремь 1. Ресничка с номером $k$ ребра $j$ уровня $i$, где $i \in \mathbf{N}_{l}$, $j \in \mathbf{N}_{2^{i-1}}$ и $k \in \mathbf{N}_{n}$, может иметь нагрузку $q_{i j k}$ такую, что $q_{i j k} \in\{0\} \cup \mathbf{N}_{2^{l-i} b}$. Значит, число всевозможных конфигураций нагрузок ребра $j$ уровня $i$ равно $\left(2^{l-i} b+1\right)^{n}$. Так как число ребер на уровне $i$ равно $2^{i-1}$, число всевозможных конфигураций нагрузок по всем ребрам уровня $i$ равно $\left(\left(2^{l-i} b+1\right)^{n}\right)^{2^{i-1}}$. Так как $I$-дерево $D^{-1}(b, r, n, l)$ имеет $l$ уровней, число всевозможных состояний этого $I$-дерева равно $\prod_{i=1}^{l}\left(2^{l-i} b+1\right)^{2^{i-1} n}$. Теорема доказана.

Утверждение теоремы 2 следует из лемм 1, 2 и 3.

Утверждение теоремы 3 следует из лемм 4, 5 и 6.

Доказательство теоремы 4. Положим $b=r$. Из теоремы 2 вытекает неравенство $|S t(b, r, n, l)| \geqslant\left|K_{s_{1}}\right|$. Так как для всякой реснички с номером $i j k$, где $i \in \mathbf{N}_{l} \backslash\{1\}$, $j \in \mathbf{N}_{2^{i-1}}$ и $k \in \mathbf{N}_{n}$, возможны $2^{l-i} b+1$ варианта ее нагрузки, для всех ресничек ребра первого уровня, кроме ресничек с номерами 111 и 112 , возможны $2^{l-1} b+1$ варианта их нагрузок, а для ресничек с номерами 111 и 112 этого ребра возможны $2^{l-1} b$ варианта их нагрузок, мощность класса $K_{s_{1}}$ равна $\left(2^{l-1} b\right)^{2}\left(2^{l-1} b+1\right)^{n-2} \prod_{i=2}^{l}\left(2^{l-i} b+1\right)^{2^{i-1} n}$. По теореме 1 число всех состояний $I$-дерева $D^{-1}(b, r, n, l)$ равно $\prod_{i=1}^{l}\left(2^{l-i} b+1\right)^{2^{i-1} n}$ и число стартовых состояний $I$-дерева $D^{-1}(b, r, n, l)$ не превосходит числа всех состояний этого $I$-дерева, поэтому справедливы оценки

$$
\frac{\left(2^{l-1} b\right)^{2}\left(2^{l-1} b+1\right)^{n-2} \prod_{i=2}^{l}\left(2^{l-i} b+1\right)^{2^{i-1} n}}{\prod_{i=1}^{l}\left(2^{l-i} b+1\right)^{2^{i-1} n}} \leqslant \frac{|S t(b, r, n, l)|}{|Q(b, n, l)|} \leqslant 1 .
$$

Отсюда получаем, что

$$
\left(\frac{2^{l-1} b}{2^{l-1} b+1}\right)^{2} \leqslant \frac{|S t(b, r, n, l)|}{|Q(b, n, l)|} \leqslant 1 .
$$


Пусть $b \geqslant 2 r$. Из теоремы 3 следует, что $|S t(b, r, n, l)| \geqslant\left|K_{m_{1}}\right|$. Мощность класса $K_{m_{1}}$ равна $2^{l-1} r\left(2^{l-1} b+1\right)^{n-1} \prod_{i=2}^{l}\left(2^{l-i} b+1\right)^{2^{i-1} n}$, так как для всякой реснички с номером $i j k$, где $i \in \mathbf{N}_{l} \backslash\{1\}, j \in \mathbf{N}_{2^{i-1}}$ и $k \in \mathbf{N}_{n}$, возможны $2^{l-i} b+1$ варианта ее нагрузки, для всех ресничек ребра первого уровня, кроме реснички с номером 111, возможны $2^{l-1} b+1$ варианта их нагрузок, а для реснички с номером 111 этого ребра возможны $2^{l-1} b-2^{l-1}(b-r)=2^{l-1} r$ варианта ее нагрузки. Так как по теореме 1 число всех состояний $I$-дерева $D^{-1}(b, r, n, l)$ равно $\prod_{i=1}^{l}\left(2^{l-i} b+1\right)^{2^{i-1} n}$ и число стартовых состояний $I$-дерева $D^{-1}(b, r, n, l)$ не превосходит числа всех состояний этого $I$-дерева, справедливы оценки

$$
\frac{2^{l-1} r\left(2^{l-1} b+1\right)^{n-1} \prod_{i=2}^{l}\left(2^{l-i} b+1\right)^{2^{i-1} n}}{\prod_{i=1}^{l}\left(2^{l-i} b+1\right)^{2^{i-1} n}} \leqslant \frac{|S t(b, r, n, l)|}{|Q(b, n, l)|} \leqslant 1 .
$$

Отсюда получаем, что

$$
\frac{2^{l-1} r}{2^{l-1} b+1} \leqslant \frac{|S t(b, r, n, l)|}{|Q(b, n, l)|} \leqslant 1 .
$$

Пусть $r<b<2 r$. Из теоремы 3 следует, что $|S t(b, r, n, l)| \geqslant\left|K_{m_{1}}\right|$. Найдем мощность класса $K_{m_{1}}$. Для всякой реснички с номером $i j k$, где $i \in \mathbf{N}_{l} \backslash\{1\}, j \in \mathbf{N}_{2^{i-1}}$ и $k \in \mathbf{N}_{n}$, возможны $2^{l-i} b+1$ варианта ее нагрузки, для всех ресничек ребра первого уровня, кроме ресничек с номерами 111 и 112, возможны $2^{l-1} b+1$ варианта их нагрузок, для ресничек с номерами 111 и 112 этого ребра возможны следующие числа вариантов их нагрузок (где через $q_{111}$ и $q_{112}$ обозначены нагрузки ресничек 111 и 112 , соответственно, в состоянии $q$, обладающем $m_{1}$-свойством):

(a) $2^{l-1}(2 r-b)-1$ для реснички с номером 111 и $2^{l-1} b$ для реснички с номером 112 (в случае $2^{l-1}(b-r)<q_{111}<2^{l-1} r$ и $q_{112}>0$ ),

(б) 1 для реснички с номером 111 и $2^{l-1} r$ для реснички с номером 112 (в случае $q_{111}=2^{l-1} r$ и $\left.q_{112}>2^{l-1}(b-r)\right)$,

(в) $2^{l-1}(b-r)$ для реснички с номером 111 и $2^{l-1} b+1$ для реснички с номером 112 (в случае $\left.q_{111}>2^{l-1} r\right)$.

Таким образом, общее число вариантов нагрузок ресничек с номерами 111 и 112 равно

$$
\left(2^{l-1}(2 r-b)-1\right) 2^{l-1} b+2^{l-1} r+2^{l-1}(b-r)\left(2^{l-1} b+1\right)=2^{l-1} r 2^{l-1} b .
$$

Из вышесказанного следует, что

$$
\left|K_{m_{1}}\right|=2^{l-1} r 2^{l-1} b\left(2^{l-1} b+1\right)^{n-2} \prod_{i=2}^{l}\left(2^{l-i} b+1\right)^{2^{i-1} n} .
$$

По теореме 1 число состояний $I$-дерева $D^{-1}(b, r, n, l)$ равно $\prod_{i=1}^{l}\left(2^{l-i} b+1\right)^{2^{i-1} n}$ и число стартовых состояний $I$-дерева $D^{-1}(b, r, n, l)$ не превосходит числа всех состояний этого $I$-дерева, поэтому справедливы оценки

$$
\frac{2^{l-1} r 2^{l-1} b\left(2^{l-1} b+1\right)^{n-2} \prod_{i=2}^{l}\left(2^{l-i} b+1\right)^{2^{i-1} n}}{\prod_{i=1}^{l}\left(2^{l-i} b+1\right)^{2^{i-1} n}} \leqslant \frac{|S t(b, r, n, l)|}{|Q(b, n, l)|} \leqslant 1 .
$$


Отсюда получаем, что

$$
\frac{\left(2^{l-1}\right)^{2} r b}{\left(2^{l-1} b+1\right)^{2}} \leqslant \frac{|S t(b, r, n, l)|}{|Q(b, n, l)|} \leqslant 1 .
$$

Теорема доказана.

Автор выражает глубокую благодарность В. Б. Кудрявцеву и А. Г. Чучалину за постановку задачи и научное руководство.

\section{Список литературы}

1. Кудрявцев В. Б., Алешин С. В., Подколзин А. С., Введение в теорию автоматов. Наука, Москва, 1985.

2. Гераськина Ю. Г., Об одной автоматной модели в биологии. Дискретная математика (2007) 19 , №3, 122-139.

3. Гераськина Ю. Г., Модель процесса дыхания живых организмов. Интеллектуальные системь (2003) 8, №1/4, 429-456.

4. Гераськина Ю. Г., Модель самоочищения легочных структур. Интеллектуальные системы (20022003) 7, №1/4, 41-54.

Статья поступила 21.03.2008. 